\title{
Reservation and Grouping Stations for the IEEE 802.11 DCF
}

\author{
Yang Xiao ${ }^{1}$, Haizhon $\mathrm{Li}^{1}$, Kui $\mathrm{Wu}^{2}$, Kin K. Leung ${ }^{3}$, and Qiang $\mathrm{Ni}^{4}$ \\ ${ }^{1}$ Computer Science Division, University of Memphis, \\ 373 Dunn Hall, Memphis, TN 38152, USA \\ yangxiao@ieee.org, hli1@memphis.edu \\ ${ }^{2}$ Department of Computer Science, University of Victoria, \\ Victoria, British Columbia, Canada V8W 3P6 \\ wkui@cs.uvic.ca \\ ${ }^{3}$ Electrical and Electronic Engineering and Computing Departments, \\ Imperial College, London SW7 2BT, United Kingdom \\ kkleung@ieee.org \\ ${ }^{4}$ Hamilton Institute, National University of Ireland, Maynooth \\ Qiang.Ni@ieee.org
}

\begin{abstract}
In this paper, we propose a novel contention-based protocol, called Reservation with Grouping Stations (RGS) for the IEEE 802.11 Distributed Coordination Function (DCF). In the proposed RGS scheme, stations are grouping into the active group, the ready group, and the idle group. Furthermore, stations announce the next frame's backoff counter beforehand. The proposed scheme is compared with the DCF and another scheme in the literature, and simulation results show that the proposed scheme is the best among these three schemes.
\end{abstract}

\section{Introduction}

The IEEE 802.11 Medium Access Control (MAC) employs a mandatory contentionbased channel access function called Distributed Coordination Function (DCF), and an optional centrally controlled channel access function called Point Coordination Function (PCF) [1]. The DCF adopts a carrier sense multiple access with collision avoidance (CSMA/CA) with binary exponential backoff. In the DCF a station with a frame to transmit monitors the channel activities until an idle period, equal to a distributed inter-frame space (DIFS), is detected. After sensing an idle DIFS, the station waits for a random backoff interval before transmitting. The backoff time counter is decremented in terms of slot time as long as the channel is sensed idle. The counter is stopped when a transmission is detected on the channel, and reactivated when the channel is sensed idle again. The station transmits its frame when the backoff time reaches zero. At each transmission, the backoff time is uniformly chosen in the range $[0, C W-1]$, where $C W$ is the current backoff window size. At the very first transmission attempt, $C W$ equals to the initial backoff window size $C W_{\min }$. After each unsuccessful transmission, $C W$ is doubled until a maximum backoff window size value $C W_{\max }$ is reached. Once it reaches $C W_{\max }, C W$ shall remain at the value $C W_{\max }$ until it 
is reset. $C W$ shall be reset to $C W_{\min }$ after every successful attempt to transmit, or the retransmission counter reaches the retry limit $L_{\text {retry. }}$ In the latter case, the frame will be dropped. After the destination station successfully receives the frame, it transmits an acknowledgment frame (ACK) following a short inter-frame space (SIFS) time. If the transmitting station does not receive the ACK within a specified ACK Timeout, or it detects the transmission of a different frame on the channel, it reschedules the frame transmission according to the previous backoff rules.

There have been many performance studies and performance enhancements for the DCF [2-14]. Calì et al. [2] studied an optimization method for a p-persistent Wireless LAN (WLAN) MAC, and proposed adaptive backoff algorithms for the p-persistent WLAN MAC [3]. Bianchi [4] proposed a simple and accurate analytical model to compute saturation throughput. Ziouva and Antonakopoulos [5] improved Bianchi's model to derive saturation delay. Bing [6] provided a performance analysis by a quantitative approach. Huang and Chen [7] gave approximate models that account for hidden terminals. Chhaya and Gupta [8] calculated the throughput of CSMA/CA using a simple model with the probabilities of capture and the presence of hidden stations. Tray and Chua [9] provided a good and approximate model for CSMA/CA. Xiao and Rosdahl $[10,11]$ identified a throughput upper limit for higher data rates and studied a group transmission and acknowledgement scheme. Leung et al. [12] studied the IEEE 802.11 MAC for outdoor cellular networks. Baldwin et al. [13, 14] proposed a real-time MAC protocol for ad hoc WLANs, in which two concepts are adopted, transmission deadline and a station's next backoff value (BV). The transmission deadline is used for selectively discarding frames which are late for their transmission deadlines at the MAC layer. A station's next BV is adopted to decrease collisions under a constant backoff window size, i.e., $8 \mathrm{~N}$ and $\left(2+\left[6 /\left(\mathrm{R}^{1 / 2}\right)\right\rfloor\right) \mathrm{N}$ in [13] and [14], respectively, where $\mathrm{N}$ is the estimated number of stations in the network and $\mathrm{R}$ is the channel data rate in Mbps, and therefore, the scheme is called Enhanced Collision Avoidance (ECA).

As indicated in the most of studies [2-9], when the number of competing stations increases, the performance dramatically decreases. In this paper, we propose a novel scheme for contention-based MAC called Reservation with Grouping Stations (RGS) to reduce collisions and improve the system performance. The fundamental reason of causing collisions in the DCF is that stations do not know other stations' information such as backoff counters so that two stations with the same backoff counter do not know that they will be definitely collided sometime later. If such information can be known beforehand, collisions may be avoided by adopting different backoff counters, and unnecessary collisions and wasted waiting time may be avoided. Therefore, in the RGS scheme, backoff counters of next frames, if available, are generated beforehand and announced in frame transmissions. We further classify stations into three groups: the active group, the ready group, and the idle group. The motivation for grouping stations is that stations in the active group do not have collisions if there are no hidden nodes since their next frames' backoff counters are known by other stations which avoid use of conflicting backoff counters. Collisions may happen among stations in the ready group since their next frames' backoff counters have not been known by 
other stations yet. The hidden node problem will be handled by Request-To-Send (RTS)/Clear-To-Send (CTS) mechanism.

This paper is organized as follows. Section 2 describes the proposed RGS scheme in detail. Performance studies are conducted in Section 3 via simulations. We conclude this paper in Section 4.

\section{Reservation with Grouping Stations}

In this section, we propose a novel scheme for contention-based MAC called Reservation with Grouping Stations (RGS).

In the RGS scheme, backoff counters of next frames, if available, are generated beforehand and announced in frame transmissions. In other words, in a station's any frame transmission, referred to as the current frame transmission, if the next frame is ready, i.e., waiting in the queue to be transmitted, the next frame's backoff counter is generated beforehand and embedded in the current frame header to announce to other stations. If the current frame is successfully transmitted, other stations know the station's next frame's backoff counter. Otherwise, other stations have no way to know the station's next frame backoff counter.

Any station denoted as S has to maintain other stations' backoff counters locally in a table, called Backoff Counter Table (BCT), shown in Fig.1a. Fig.1a shows S's BCT, in which S's own backoff counter is 4 , and backoff counters of stations A, B, and D are 6,2 , and 9, respectively. Station $C$ had already transmitted its previous frame which does not include its next frame backoff information, and we use -1 to indicate the case that the next frame's backoff counter is not available. Whenever $\mathrm{S}$ hears an announcement of the next frame's backoff counter of another station denoted as F, S stores/updates F's backoff counter in S's local BCT. whenever S's backoff counter is decreased due to the channel access rule described in introduction such as detecting an idle slot, other stations' backoff counters in S's BCT are also decreased; whenever S's backoff counter freezes when detecting a busy channel, other stations' backoff counters in S's local table also freeze. Before S transmits the current frame, if it has the next frame waiting in the queue, it generates a random backoff counter for the next frame and embedded it in the current frame's MAC header to announce to other stations. If $S$ is in the ready state defined in the next, whenever it receives a frame of the station $\mathrm{F}$ indicating that $\mathrm{F}$ has chosen the same backoff counter, $\mathrm{S}$ needs to choose another backoff counter since a collision will certainly occur.

Furthermore, as shown in Fig.1b, a station $\mathrm{S}$ has three states: active, ready, and idle. $\mathrm{S}$ is in the idle state if it has no frame ready to transmit. $\mathrm{S}$ is in the active state if it has a frame ready to transmit and this frame's backoff counter has been successfully announced through the previous successfully transmitted frame so that other stations know this information. $\mathrm{S}$ is in the ready state if it has a frame ready to transmit, but this frame's backoff counter has not been successfully announced to other stations. All the stations in the active state, the ready state, and the idle state form an active group, a ready group, and an idle group, respectively. We explain S's state diagram in Fig.1b as follows. 


\begin{tabular}{|l|c|c|c|c|c|c|}
\hline Stations & Self & A & B & C & D & $\ldots$ \\
\cline { 1 - 5 } Counters & 4 & 6 & 2 & -1 & 9 & $\ldots$ \\
\hline
\end{tabular}

(a) Backoff Counter Table (BCT)

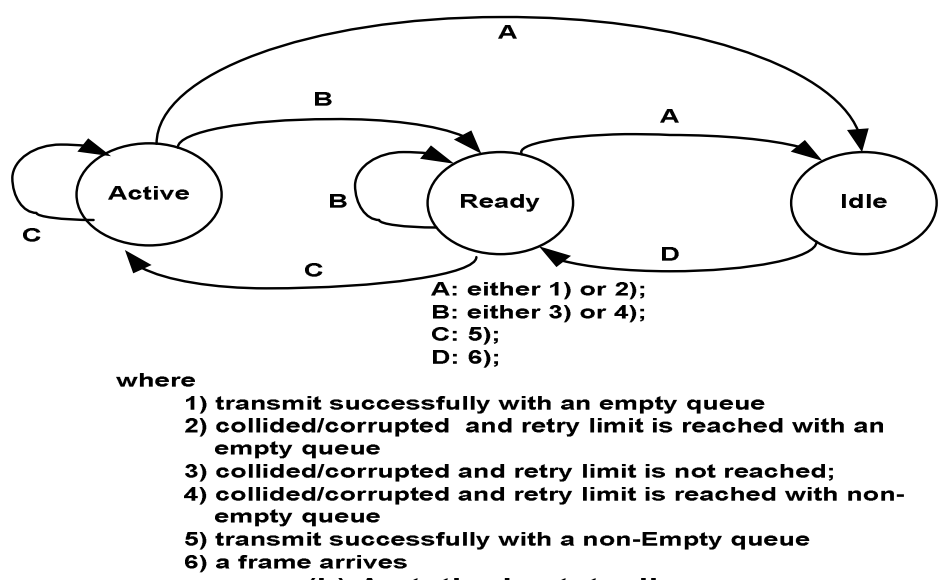

(b) A station's state diagram

Fig. 1. The state diagram of a station $S$

a) $\mathrm{S}$ in the idle state changes to the ready state when a frame arrives (condition D);

b) $\mathrm{S}$ in the ready state changes to the active state if a frame, denoted as the current frame, is transmitted successfully and there is another frame, denoted as the next frame, in the queue so that the next frame's backoff is generated beforehand and announced through the current frame's transmission;

c) $\mathrm{S}$ in the ready state remains in the ready state if the current frame transmission is collided/corrupted and the retry limit is not reached;

d) $\mathrm{S}$ in the ready state remains in the ready state if the current frame transmission is collided/corrupted, the retry limit is reached, and there is another frame in the queue;

e) $\mathrm{S}$ in the ready state changes to the idle state if the current frame transmission is collided/corrupted, the retry limit is reached, and there is not another frame in the queue;

f) $\mathrm{S}$ in the ready state changes to the idle state if the current frame transmission is transmitted successfully and there is not another frame in the queue;

g) $\mathrm{S}$ in the active state remains in the active state if a frame is transmitted successfully and there is another frame in the queue;

h) $\mathrm{S}$ in the active state changes to the ready state if the current frame transmission is collided/corrupted and the retry limit is not reached;

i) $\mathrm{S}$ in the active state changes to the ready state if the current frame transmission is collided/corrupted, the retry limit is reached, and there is another frame in the queue; 
j) $\mathrm{S}$ in the active state changes to the idle state if the current frame transmission is collided/corrupted, the retry limit is reached, and there is not another frame in the queue;

k) $\mathrm{S}$ in the active state changes to the idle state if the current frame transmission is transmitted successfully and there is not another frame in the queue;

Stations in the active group do not have collisions if there are no hidden nodes since their next frames' backoff counters are known by other stations which avoid using conflicted backoff counters. The hidden node problem can be reduced if $\mathrm{Re}-$ quest-to-Send (RTS)/Clear-to-Send (CTS) mechanism is adopted. Collisions may happen among stations in the ready group since their next frames' backoff counters have not been known by other stations yet. If the hidden nodes exist, active stations may get collisions and in this case, the station will change from the active state into the ready state, as shown in Fig.1.

The next frame's backoff counter is chosen uniformly and randomly from the set $\left\{a_{1}, a_{2} \ldots, a_{n}\right\}$, where $a_{1}<a_{2}<\ldots<a_{n}$ such that for any integer $m$ where $a_{n} \geq m \geq 0$, we have either $m \in\left\{a_{1}, a_{2} \ldots, a_{n}\right\}$ or $m$ equals to one of other stations' backoff counters in the station's BCT table. In other words, the set includes the next $n$ available/unreserved slots. The purpose of the above choices is to avoid choosing conflicted slots already reserved by other stations.

Let $N_{l}, N_{2}$, and $N_{3}$ denote the numbers of stations in the active group, the ready group, and the idle group, respectively.

We propose an approach to estimate the value of $N_{2}$. Each station measures its activities during three states as follows. Let $T_{1}(j), T_{2}(j)$, and $T_{3}(j)$ denote the portions of time spending in the active state, the ready state, and the idle state, respectively, for the station $j$. We have: $T_{1}(j)+T_{2}(j)+T_{3}(j)=1$. Whenever station $j$ transmits a frame, it piggybacks the $T_{1}(j)$ and $T_{2}(j)$ values. Note that the $T_{3}(j)$ value can be derived based on the $T_{1}(j)$ and $T_{2}(j)$ values. Therefore, other stations also know them $\left(T_{1}(j), T_{2}(j)\right.$, and $\left.T_{3}(j)\right)$ and periodically update their values for station $j$. Let $M(\Delta t, t)$ denote the number of piggybacked frames from different stations obtained during the time period from $t-\Delta t$ to $t$, where $\Delta t$ is a relative large value. Multiple frames from the same station count only once, i.e., the most current one. Then, current measured $N_{2}(t)$ value can be approximated by

$$
N_{2, \text { current }}(t)=\sum_{j=1}^{M(\Delta t, t)} T_{2}(j)
$$

Note that some idle stations may not necessarily send the piggyback frames, but this information does not have impacts on estimation of the value $N_{2}$. Let $t^{\prime}$ denote the previous measurement time, and $\alpha(0 \leq \alpha \leq 1)$ denote a weight value. An exponential smoothing technique can be used to obtain estimated $N_{2}(t)$ value as follows.

$$
N_{2}(t)=\alpha N_{2}\left(t^{\prime}\right)+(1-\alpha) N_{2, \text { current }}(t)
$$

In the proposed RGS scheme, the original binary exponential backoff is still used. However, the initial window size is chosen as $2\left(N_{l}+N_{2}\right)$. 


\section{Simulation Results}

In the simulations, we adopt the IEEE 802.11a as an example, and we compare the proposed scheme with the DCF and the ECA scheme in [13-14] in terms of average throughput, instant throughput, access delay, and the number of collisions.

Parameters are chosen as the default values in the IEEE 802.11a. The control rate is $24 \mathrm{Mbps}$ and the data rate $54 \mathrm{Mbps}$. Beacon interval is $100 \mathrm{~ms}$. The mean of frame sizes is 300 bytes. Each station has traffic with ON-OFF periods. Initially there are 3 stations in the system. After each 5 seconds, 3 stations join the system until total 60 stations in the system. Each station operates in ON/OFF states alternatively. The times spent in the ON and OFF states are exponentially distributed with a mean of 3 seconds and 6 seconds, respectively. In the ON state, the station's queue is never empty. In the OFF state, the station has nothing to transmit. The total simulation time is $300 \mathrm{~s}$.

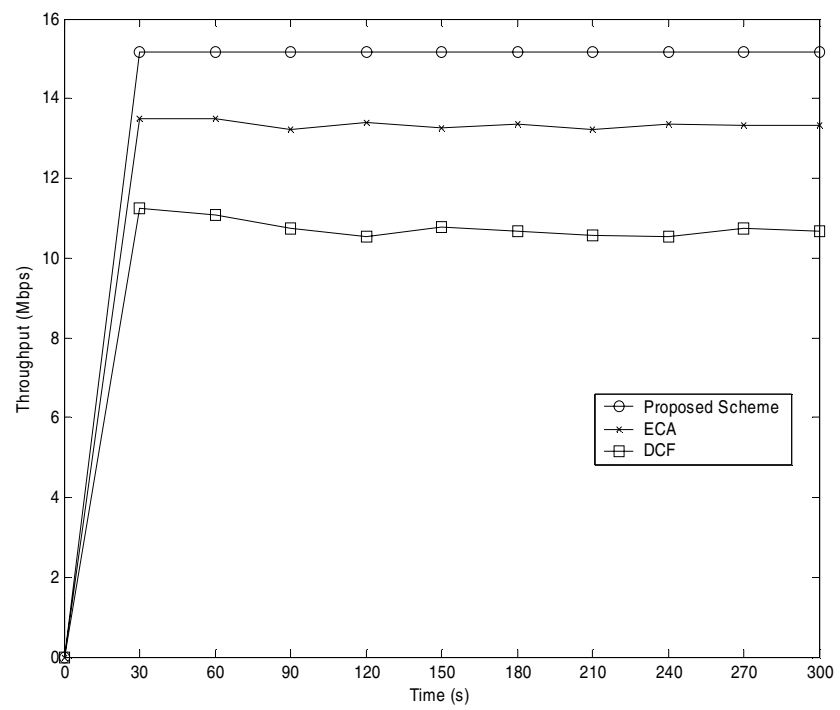

Fig. 2. Average Throughput (Mbps)

Fig. 2 compares the average throughputs of the DCF, ECA, and the proposed scheme. As illustrated in the figure, both the proposed scheme and the ECA outperform the DCF. The proposed scheme is the best among the three approaches in terms of the average throughput.

Fig. 3, Fig. 4, and Fig. 5 show the instant throughputs of the DCF, ECA, and the proposed scheme, respectively, where the instant throughput is defined as the throughput during a very short interval (per beacon interval), whereas the average throughput is defined as the throughput during a much larger interval. We observe that throughputs in the DCF and the ECA fluctuate a lot, whereas in the proposed scheme, throughput is much stable. 


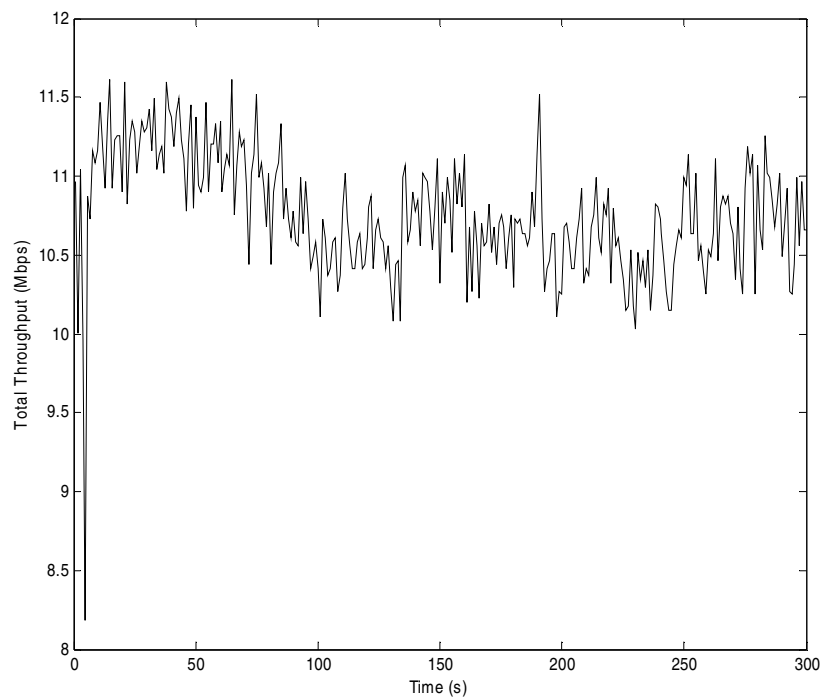

Fig. 3. Instant Throughput of the DCF (Mbps)

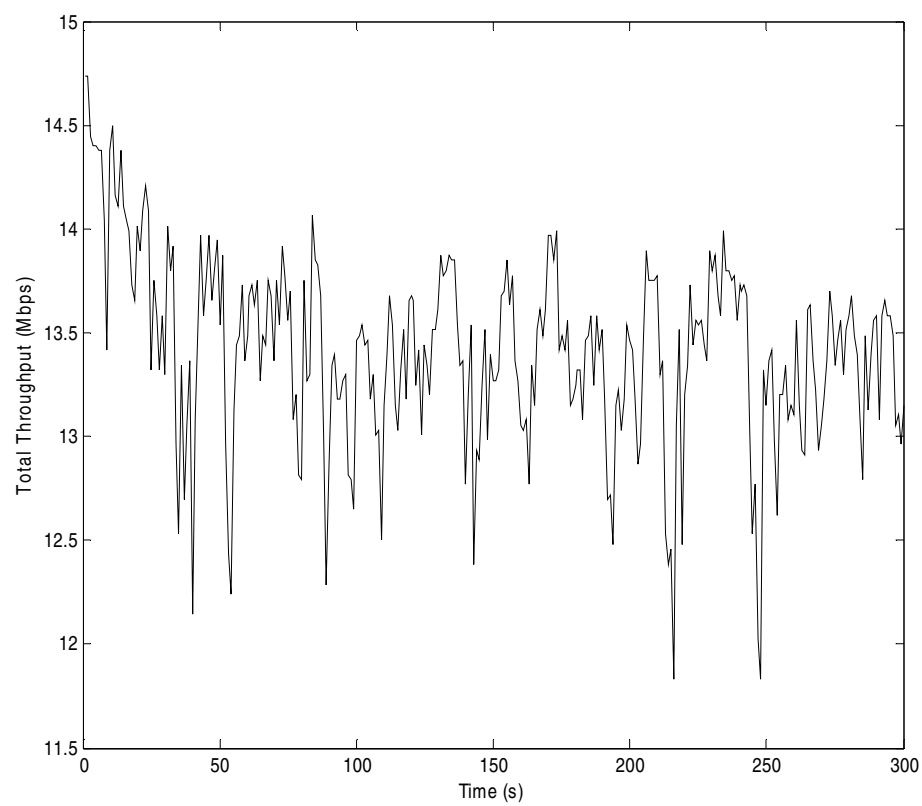

Fig. 4. Instant Throughput of the ECA (Mbps)

Fig. 6 and Fig. 7 show the numbers of collisions. As illustrated in Fig. 6, both the proposed scheme and the ECA have small numbers of collisions and are better than the DCF. The DCF in facts has a large number of collisions. Fig. 7 compares the proposed 


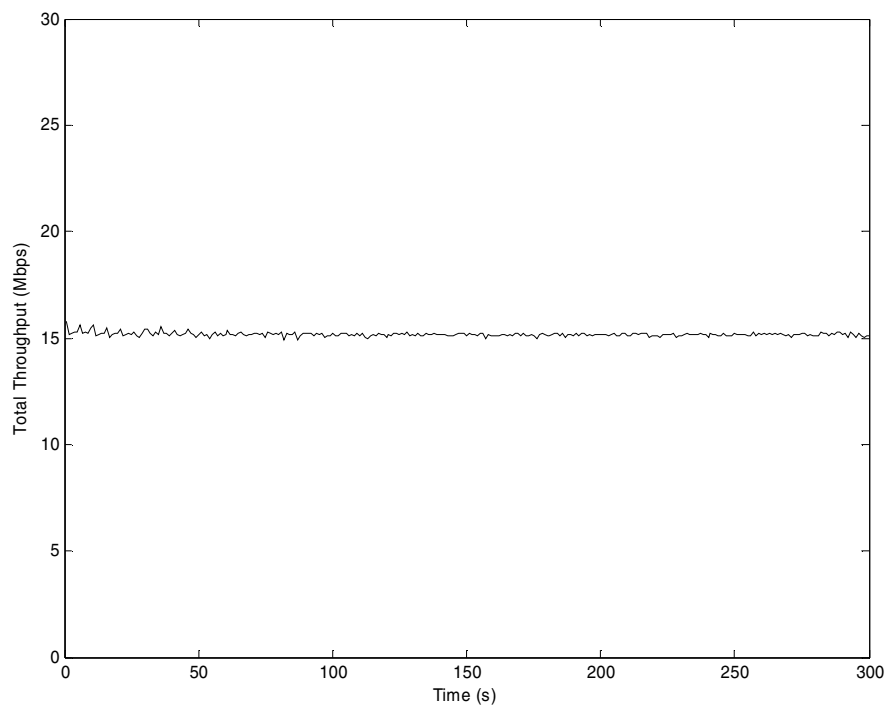

Fig. 5. Instant Throughput of the proposed scheme (Mbps)

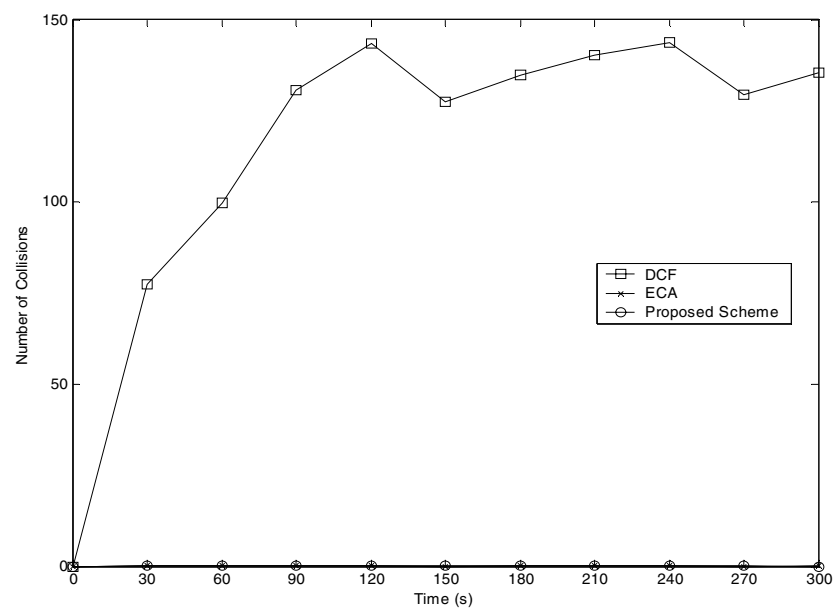

Fig. 6. Number of Collisions

scheme with the ECA, and shows that the ECA has less frequent collisions but each time with a relatively higher number of collisions than the proposed scheme, whereas the proposed scheme has more frequent collisions but each time with a small number of collisions.

At this moment, readers may wonder that since the ECA's number of collisions is not very bad, why it has a worse throughput performance than the proposed scheme. The answer is that the proposed scheme reduces a lot of empty slots than the ECA. 

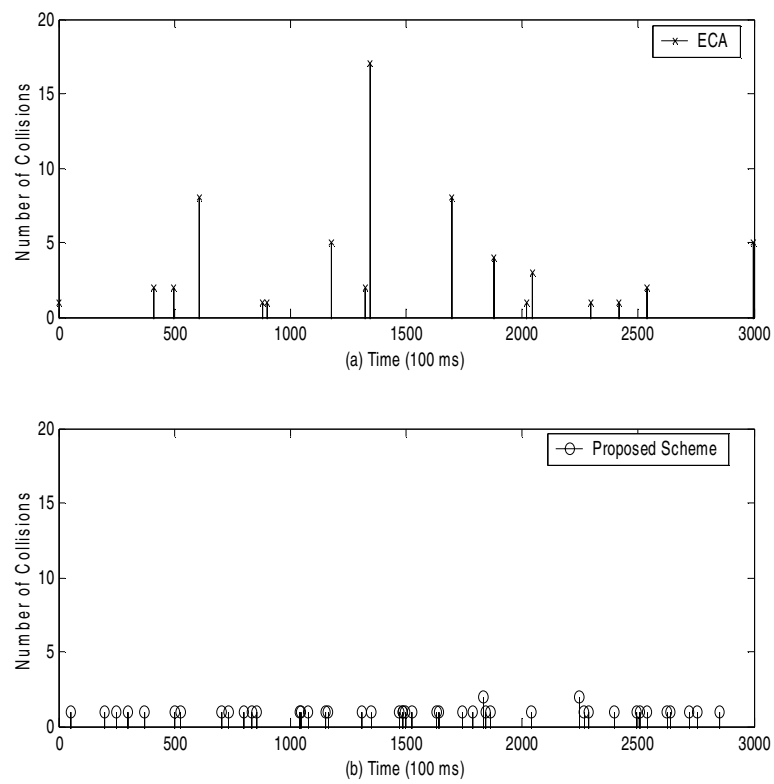

Fig. 7. Number of Collisions

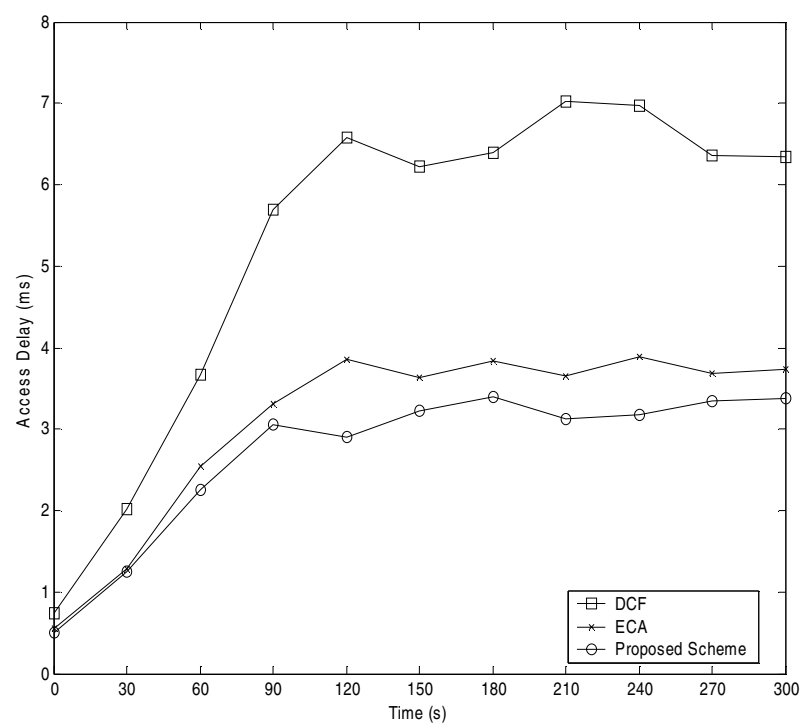

Fig. 8. Access Delay

Fig. 8 shows the access delays of all three schemes, where the access delay is defined as the time interval between when a station is ready to transmit a frame (the frame is in head of the waiting queue) and when the frame has been successfully transmitted. As illustrated by the figure, both the proposed scheme and the ECA outperform the DCF, and the proposed scheme is the best. 


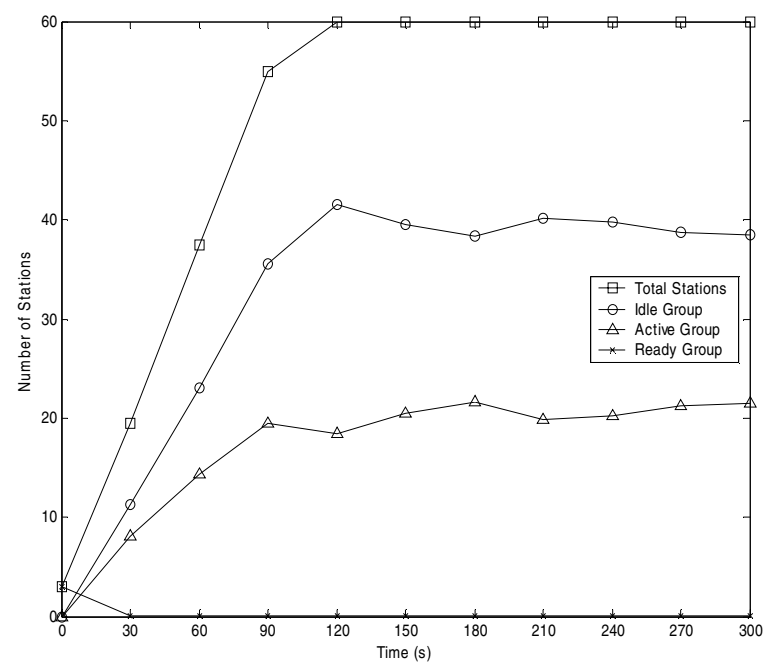

Fig. 9. Grouping

Fig. 9 shows the number of stations in the active, ready, and idle groups of the proposed scheme in this simulation setup. It shows that the number of stations in the ready state is pretty small, but not zero. The numbers of stations in the active group and the idle groups are relatively large.

In fact, we also perform simulations in many other situations such as when the number of stations in the ready group is large too, and we also have similar conclusions. We omit the results due to the limited space.

\section{Conclusion}

This paper proposed a novel contention-based protocol based on the concept of grouping stations as well as announcing the backoff counters in advance. Simulation studies were performed to compare with the new protocol with the DCF and ECA, and our results show that the proposed scheme is the best in terms of throughput and delay. The proposed scheme outperforms the DCF and ECA because: 1) announcing the backoff counters in advance avoids possible collisions among active stations; and 2) grouping stations enable one to estimate the number of ready stations, thus determining the appropriate window size to reduce collisions among ready stations.

\section{References}

1. IEEE 802.11: Wireless LAN Medium Access Control (MAC) and Physical Layer (PHY) specification, 1999

2. F. Calì, M. Conti, and E. Gregori, "Dynamic Tuning of the IEEE 802.11 Protocol to Achieve a Theoretical Throughput Limit," IEEE/ACM Trans. Networking, Vol. 8, No. 6, Dec. 2000, pp. 785-790. 
3. F. Cali, M. Conti, and E. Gregori, "IEEE 802.11 Protocol: Design and Performance Evaluation of an Adaptive Backoff Mechanism,” IEEE J-SAC, Vol. 18, No. 19, Sep. 2000, pp. 1774-1786.

4. G. Bianchi, "Performance Analysis of the IEEE 802.11 Distributed Coordination Function," IEEE J-SAC Vol. 18 No. 3, Mar. 2000, pp. 535-547.

5. E. Ziouva and T. Antonakopoulos, "CSMA/CA performance under high traffic conditions: throughput and delay analysis," Computer Communications, 25 (2002), pp.313-321.

6. B. Bing and R. Subramanianb, "A novel technique for quantitative performance evaluation of wireless LANs," Computer Communications, Vol. 21, No. 9, July 1998, pp.833-838.

7. K. C. Huang and K.-C. Chen, "Interference analysis of nonpersistent CSMA with hidden terminals in multicell wireless data networks", in Proc. IEEE PIMRC, Toronto, Canada, Sept. 1995, pp.907-911.

8. H. S. Chhaya and S. Gupta, "Performance modeling of asynchronous data transfer methods of IEEE 802.11 MAC protocol", Wireless Networks, vol. 3, pp. 217-234, 1997.

9. Y. C. Tay and K. C. Chua, "A Capacity Analysis for the IEEE 802.11 MAC Protocol," Wireless Networks 7, 2001, pp. 159-171.

10. Y. Xiao and J. Rosdahl, “Throughput and Delay Limits of IEEE 802.11,” IEEE Communications Letters, Vol. 6, No. 8, Aug. 2002, pp. 355-357.

11. Y. Xiao and J. Rosdahl, "Performance Analysis and Enhancement for the Current and Future IEEE 802.11 MAC Protocols," ACM SIGMOBILE Mobile Computing and Communications Review (MC2R), Vol. 7, No. 2, Apr. 2003, pp. 6-19.

12. K. K. Leung, B. McNair, L.J. Cimini, and J.H. Winters, "Outdoor IEEE 802.11 Cellular Networks: MAC Protocol Design and Performance," Proc. of IEEE ICC 2002, New York, April 2002.

13. R. O. Baldwin, N. J. Davis IV, and S. E Midkiff, "A real-time Medium Access Control Protocol for Ad hoc Wireless Local Area Networks," Mobile Computing and Communications Review (MC2R), Vol. 3, No. 2, pp. 20-27, Apr. 1999.

14. R. O. Baldwin, N. J. Davis IV, S. E. Midkif, and R. A. Raines, "Packetized Voice Transmission using RT-MAC, a Wireless Real-time Medium Access Control Protocol," Mobile Computing and Communications Review (MC2R), Vol. 5, No. 3, pp. 11-25, Jul. 2001.

15. IEEE 802.11a: High-speed Physical Layer in the 5GHz Band, 1999. 